\title{
PERCEIVED EFFort of FunCTIONAL Activities AFter a Period of Mechanical Ventilation
}

\begin{abstract}
A case report to assess exercise testing post-mechanical ventilation, using functional activities while monitoring changes in the physiological parameters and subjective rating of perceived exertion. The case report subject was able to use the Modified Borg Scale to report her perception of perceived exertion during functional exercise testing. An increase in heart rate, respiratory rate and Modified Borg Scale rating was noted on progression through the exercise test. Difficulty was experienced with subject follow-up due to discharged from hospital as soon as the patient was considered to be medically stable. The subject was unable to complete the functional exercise test before discharge.
\end{abstract}

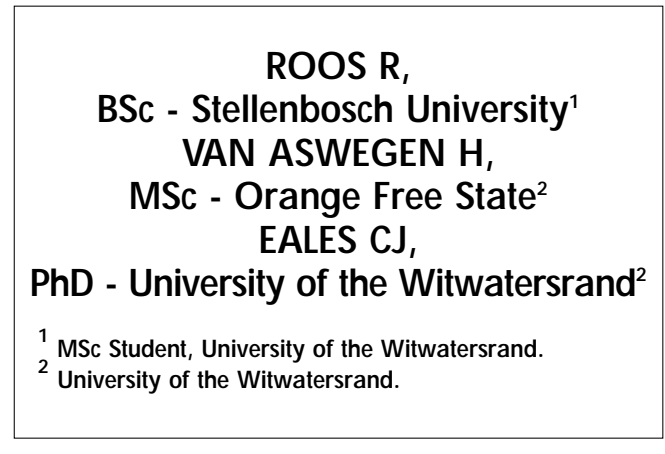

KEY WORDS: MECHANICAL VENTILATION, EXERCISE, PERCEIVED EXERTION.

\section{INTRODUCTION}

Physiotherapists working in intensive care units (ICU), provide chest physiotherapy and early rehabilitation to patients recovering from critical illnesses. As a result, they should have an impact on the overall functional ability of these patients. The objective testing of physical fitness and the ability to exercise after a period of mechanical ventilation, are difficult to assess in the critical care setting when using routine tests. Functional exercise tests such as the six-minute walking test, shuttle walking test or step test are inappropriate and not possible at this early stage of rehabilitation. An exercise test needs to be able to assess the desired functional outcome in the acute care setting. It also needs to meet the activity levels of the patient population being tested.

By using functional activities such as moving from supine to side lying, to sitting over the edge of the bed, to standing, to transferring into a chair and lastly marching on the spot, it appears possible to assess the exercise ability of these patients. By monitoring physiological parameters and the subjective rating of perceived exertion, the cardiopulmonary response to physical stress could be assessed. It appears that there are no studies reporting on subjective rating of perceived exertion during functional activities in the early rehabili- tation phase in ICU, after mechanical ventilation.

Performing functional activities independently requires great effort on the patients' part when recovering from critical illnesses. They usually report fatigue, muscle weakness, dyspnoea or difficulty in breathing during or after activity. The patient's experience when undergoing physical activity can be monitored by including the subjective rating of his/her perceived exertion. At the same time the objective aspects of physical strain when performing functional activities by individuals that are recovering from a critical illness can also be monitored.

Borg (1970) who developed the perceived exertion scales stated: "Since man reacts to the world as he perceives it and not as it really is, it is important to know more about the relation between objective and subjective measurements of physical stress". Rating perceived exertion integrates signals from peripheral working muscles and joints, central cardiovascular and respiratory function, and from the central nervous system. All these signals and experiences are integrated to form the "Gestalt" of perceived exertion. Borg (1982) was of the opinion that perceived exertion was the single best indicator of the degree of physical strain.

The Modified Borg Scale (MBS) is an ordinal scale aligned vertically with discrete numerical divisions from 0 to 10. Most, but not all of the numerical intervals on the MBS are marked with verbal descriptions of graded intensity. The scale range from "nothing at all" up to "very, very heavy/strong" when rating perceived exertion. Limited information is available regarding the use of the MBS in the acute care setting.

Lush et al (1988) used the MBS in their study to assist mechanically ventilated patients with pulmonary disease in quantifying the severity of their dyspnoea. They attributed the onset of dyspnoea to environmental variables such as suctioning, turning, weighing, position changes, sitting up in a chair and physiotherapy. The researchers suggested that it was important for clinical nurses and researchers to measure the patient's perception of dyspnoea rather than to rely on physiological or environmental parameters.

In a case study Jacovone and Young (1998) described the effectiveness of the

\section{CORRESPONDENCE TO:}

\section{Ronel Roos}

Physiotherapy Department

7 York Road,

Parktown, 2193

Johannesburg

South Africa

Tel: (011) 717-3702 
MBS in assisting a difficult-to-wean patient in quantifying her dyspnoea. The patient's difficulty with dyspnoea on exertion during physiotherapy was addressed by instructing her to avoid holding her breath and to exhale during position change from sitting to standing. She was also encouraged to give feedback by using the MBS during these activities. These interventions gave the patient hope and a sense of improved well-being, which contributed to her successful weaning from the ventilator.

Kendrick et al (2000) used the MBS in an emergency department, to enable patients with chronic obstructive pulmonary disease (COPD) and acute bronchospasm to assess the improvement of their dyspnoea levels after inhalation therapy. The improvement in MBS scores correlated with improvement in the oxygen saturation and peak expiratory flow rates. Patients gave the MBS a high satisfaction rating on ease of use. After the study the triage respiratory assessment notes and nursing notes were streamlined to include assessment on the MBS, oxygen saturation and peak expiratory flow rates.

Rating of perceive exertion is more commonly used in cardiac and pulmonary rehabilitation. The American Association of Cardiovascular and Pulmonary Rehabilitation (1999) includes the Borg Scales for rating perceived exertion in their guidelines for cardiac rehabilitation and secondary prevention programs.

Fitts and Guthrie (1995) assessed the exercise ability of patients with chronic renal failure during a six minute walking test. Subjective physical effort was rated using the MBS. This exercise testing method was used to monitor change in subjective effort, while assessing changes in heart rate during distance walked.

Grant et al (1999) determined which subjective scale, the Visual Analogue Scale (VAS), the MBS or the Likert Scale, was more reproducible and sensitive to change in the assessment of breathlessness and general fatigue during sub-maximal exercise testing in a group of active male volunteers. The MBS was most sensitive in assessing change in general fatigue.

In each of the above-mentioned studies the subjects were able to use the MBS to report their symptoms. The motivation for using the MBS during functional exercise testing is to include the subjective perception of physical strain. This information could improve the researcher's understanding of what patients experience during functional activities when recovering from a critical illness.

\section{METHOD}

Consent was obtained from the management of the hospital where the patient was being treated as well as from the caring physicians. Informed consent was obtained from the patient.

\section{Inclusion Criteria}

The inclusion criteria was that a patient had to:

- Be admitted to an intensive care unit

- Be orientated with a Glascow Coma Scale of 15/15,

- Be between the ages of 18 - 80 years old; and

- Have received mechanical ventilation for five days or longer.

\section{Exclusion Criteria}

Since the patient had to give subjective feedback and had to be able to mobilized out of bed, the exclusion criteria included:

- Mentally handicapped individuals

- Patients with head injuries/spinal cord injuries/unstable pelvis fractures/ unstable vertebral fractures

- Pre-admission history of dementia/ confusion/Alzheimer's disease and illiterate subjects.

A review of the patient's chart and a general assessment were carried out prior to exercise testing to determine the severity of illness and prior level of functioning.

The exercise test consisted of the following functional activities; supine (trunk elevated 30 degrees) to side lying, side lying to sitting over the side of the bed, sitting to standing, transferring from the bed to a chair and walking for a period of sixty seconds. The patient could use an assistive device such as a bedrail or walker during the test, but had to complete each stage independently without any outside manual support. If the patient was unable to complete a stage independently the researcher stopped the test. The patient could also stop the test at any time due to physical discomfort or to psychological reasons.

During the exercise test physiological variables such as heart rate (HR), respiratory rate (RR) and oxygen saturation $\left(\mathrm{SpO}_{2}\right)$ were measured at the end of each stage. Blood pressure (BP) and peak flow (PEFR) were measured at baseline, sitting over the edge of the bed, sitting out in a chair and after walking. At the end of each stage the patient had to report the perception of their physical effort/exertion as rated on the Modified Borg Scale (MBS). At each assessment session baseline measures were documented for physiological variables as well as the rating on the MBS of the effort of breathing prior to the start of the test. The equipment used during testing included the following: stopwatch, walker, Wright Peak Flow Meter with disposable mouth piece, oxygen saturation monitor, sphygmomanometer, noninvasive blood pressure cuff and electro cardio grams (ECG) when patients was still in the intensive care unit.

\section{CASE HISTORY}

The subject was a 67 year old lady admitted for coronary artery bypass grafting. Immediately after surgery she was admitted to a surgical intensive care unit for close monitoring due to cardiovascular system (CVS) instability. This instability was addressed by adrenaline infusions. She received ventilator support via an endotracheal tube. Weaning from the ventilator was a slow process, and the route of ventilation was changed to a tracheostomy tube two weeks after admission. She developed bilateral pleural effusions and this condition was rectified by insertion of two intercostals drains. Five days after insertion of the tracheostomy tube, the patient had difficulty breathing due to an air leak, and she was re-intubated with an endotracheal tube. After 26 days of ventilatory support she was successfully extubated. She consented to participation in this study four days after having been extubated. At this time she was orientated to person and place with a GCS score of $15 / 15$. Her vital signs were stable: 


\begin{tabular}{|l|l|}
\hline HR & 96 \\
\hline $\mathbf{R R}$ & 24 \\
\hline $\mathbf{S p O}_{\mathbf{2}}$ & $95 \%$ \\
\hline $\mathbf{B P}$ & $150 / 80$ \\
\hline Temperature & $36 \mathrm{EC}$ \\
\hline
\end{tabular}

She received two litres of oxygen via nasal cannulae. Auscultation of her chest revealed bilateral decreased air entry to the bases of the lungs with no other upnormal breath sounds. She had a strong unproductive cough. She underwent exercise testing two times per week for the duration of two weeks. The testing was discontinued when the patient was discharged from hospital. (Refer to table one for case subject characteristics and table two for exercise testing results).

\section{DISCUSSION}

A patient's exercise ability is determined by the functional aerobic capacity of the individual. Monitoring objective parameters during exercise testing provide information regarding the patient's tolerance for exercise. When rating perceived exertion during activity, the patient's perception regarding his/her physical effort is quantified. By including the objective and subjective aspects of physical activity, a more holistic approach to monitoring the functional exercise ability of the individual can be achieved. The information obtained can also be used to evaluate the effect of medical and rehabilitation intervention.

The patient population the researcher was interested in, had been admitted to an intensive care unit, and had undergone prolonged mechanical ventilation. The reason for assessing the patient at this stage, was to determine the subjective perception of the physical effort when performing functional activities. The patient was assessed after the discontinuation of mechanical ventilation. At this stage, the critical period of her illness had passed and focus was more on rehabilitation. The deconditioning effect of bed rest is well documented and are:

- A decrease in physical work capacity,

- A decrease in lung volumes and muscle strength; and

Table 1: Case Subject Characteristics

\begin{tabular}{|l|l|}
\hline Age & 67 years old \\
\hline Sex & Female \\
\hline Diagnosis & $\begin{array}{l}\text { Coronary artery bypass grafting with } \\
\text { CVS instability }\end{array}$ \\
\hline Mechanical Ventilation (days) & 26 days \\
\hline Complications in ICU & $\begin{array}{l}\text { Difficulty weaning, bilateral pleural effusions } \\
\text { and tracheostomy air leak. }\end{array}$ \\
\hline Prior level of activity & $\begin{array}{l}\text { Independent with regard to activities of } \\
\text { daily living }\end{array}$ \\
\hline Occupation & Pensioner \\
\hline Past Medical History & Arthritis left knee and hypertension. \\
\hline
\end{tabular}

Table 2: Exercise Testing 0 utcomes

\begin{tabular}{|c|c|c|c|c|c|c|}
\hline & MBS & $H R$ & BP & $\mathbf{R R}$ & PEFR & $\mathrm{SpO}_{2}$ \\
\hline \multicolumn{7}{|l|}{ Session 1} \\
\hline Baseline & 5 & 81 & $164 / 91$ & 15 & 80 & $96 \%$ \\
\hline Turning & 5 & 87 & & 22 & & $98 \%$ \\
\hline \multicolumn{7}{|l|}{ Session 2} \\
\hline Baseline & 5 & 86 & $129 / 67$ & 14 & 200 & $99 \%$ \\
\hline Turning & 6 & 116 & & 24 & & $97 \%$ \\
\hline \multicolumn{7}{|l|}{ Session 3} \\
\hline Baseline & 0 & 80 & $120 / 70$ & 26 & 170 & $90 \%$ \\
\hline Turning & 0 & 83 & & 28 & & $84 \%$ \\
\hline Sitting & 2 & 85 & $120 / 70$ & 28 & 260 & $87 \%$ \\
\hline Standing & 8 & 91 & & 32 & & $92 \%$ \\
\hline \multicolumn{7}{|l|}{ Session 4} \\
\hline Baseline & 0.5 & 85 & $130 / 70$ & 28 & 210 & $90 \%$ \\
\hline Turning & 0.5 & 77 & & 28 & & $94 \%$ \\
\hline Sitting & 0.5 & 86 & & 32 & 250 & $92 \%$ \\
\hline Standing & 1 & 85 & & 28 & & $97 \%$ \\
\hline Transfer & 9 & 84 & $120 / 80$ & 36 & 190 & $96 \%$ \\
\hline
\end{tabular}

- An increase in heart rate response on effort (O’Sullivan 1994).

Knowing the deconditioning effects of bed rest during a critical illness, close monitoring of this population during physical activity is required.

The subject was able to use the Modified Borg Scale (MBS) to report her perceived exertion during functional exercise testing. She reported the rating by verbalizing feedback, and pointing with a finger to the level on the MBS.

Initially she had difficulty reading the scale, due to weak eye sight. This problem was overcome by enlarging the lettering and numbers. She was then able to report her effort after activity.

The exercise testing method was appropriate for the subject's functional ability. At this stage of rehabilitation focus is on increasing independence with bed mobility, transfers and walking. The subject was unable to complete the exercise testing the first session. She was only able to move from supine to side lying independently in the first session. On the day of discharge, she was able to transfer from her bed to a chair independently (two weeks after discontinuation of mechanical ventilation). 
She was unable to walk after the transfer due to fatigue. The researcher found it alarming that the subject was discharged before being able to walk independently. A reason for this could be the eagerness of the subject to be discharged as soon as possible. The excessive medical cost of prolonged hospitalisation could have contributed to this early discharge.

When looking at the rating of perceived exertion results and physiological parameters, there seem to be a trend. When moving from supine to a higher functional activity, the heart rate response and respiratory rate increased as did the perception of physical effort. In previous research conducted by Grant et al (1999) the ratings from the Modified Borg Scale correlated high with heart rate and respiratory rate during sub-maximal exercise.

The peak flow readings increased when moving from supine to the higher functional activity in the exercise test.
This could be explained by improved biomechanical use of abdominal and intercostal muscles when sitting upright compared to lying down.

The only exception was noted during the fourth sessions. Whilst transferring from bed to chair the patient's peak flow measure was decreased from $250 \mathrm{l} / \mathrm{min}$ to $190 \mathrm{l} / \mathrm{min}$. She rated her perception of effort at $9 / 10$ and was unable to continue with the exercise test due to fatigue. In a study conducted by Kendrick et al (2000) they demonstrated that an improvement in MBS rating correlated with improvement in peak flow readings and oxygen saturation. However the improvement in dyspnoea was due to effect of inhalation therapy on reducing the acute bronchospasm. In this case study the peak flow readings were used to give information regarding respiratory muscle strength rather than the presence of bronchospasm.

The researcher set out to determine if it was possible to measure the physical effort (subjective and objective) of patients in an acute setting when performing functional activities. Cardiopulmonary responses to activity would always have to be monitored during the critical illness stage in any case, but it seemed beneficial to include the Modified Borg Scale during the exercise testing sessions. The patient's perception of physical effort seems to be a realistic way of judging the exercise effort.

One potential problem during functional exercise testing was identified. This was that the physical ability of a patient could be so severely limited that he/she could not turn onto his/her side independently. This could be overcome by using a more simple functional activity such as ankle plantar-flexion and dorsi-flexion for a set number of repetitions as stage one. One could then progress to turning from supine to side-lying.

The researcher would like to thank the management of Sunninghill Hospital for their permission to conduct this study.

\section{REFERENCES}

American Association of Cardiovascular and Pulmonary Rehabilitation 1999 Guidelines for Cardiac Rehabilitation and Secondary Prevention Programs, 3rd edition. Human Kinetics Publishers, Inc: $62-64$

Borg G 1970 Perceived exertion as an indicator of somatic stress. Scandinavian Journal of Rehabilitation Medicine 2(3): 92 - 98

Borg G 1982 Psychophysical bases of perceived exertion. Medicine and Science in Sport and Exercise 14: 377 - 381

Fitts S, Guthrie M R 1995 Six minute walk by people with chronic renal failure: assessment of effort by perceived exertion. American Journal of Physical Medicine and Rehabilitation 74: $54-58$
Grant S, Aitchison T, Henderson E, Christie J, Zare S, McMurray J, Dargie H 1999 A comparison of the reproducibility and the sensitivity to change of visual analogue scale, Borg scale and Likert scale in normal subjects during sub-maximal exercise. Chest 116: $1208-1217$

Jacavone J, Young J 1998 Use of pulmonary rehabilitation strategies to wean a difficultto-wean patient: case study. Critical Care Nursing 18: 29 - 37

Kendrick K R, Baxi S C, Smith R M 2000 Usefulness of the modified 0 - 10 Borg scale in assessing the degree of dyspnea in patient with COPD and asthma. Journal of emergency Nursing 26: 216 - 222
Lush M T, Jason-Bjerklie S, Carrieri V K, Lovejoy N 1988 Dyspnea in the ventilator assisted patient. Heart and Lung 17: 528 - 535

O'Sullivan S B 1994 Coronary Artery Disease. In: Physical Rehabilitation Assessment and Treatment. o'Sullivan S, Schmitz T J (editors), 3rd edition. F A Davis Company, Philadelphia: 302 